\title{
Numerical Simulation of Flow Around Railway Vehicle in Turbulent Boundary Layer over Flat Terrain
}

\author{
Koji NAKADE \\ Vehicle Aerodynamics Laboratory, Environmental Engineering Division
}

\begin{abstract}
In order to investigate the running effects on aerodynamic characteristics of a railway vehicle under strong cross winds, the author performed Large-Eddy Simulation of flow around a simple running train model. To simulate the cross winds which affect a running train, the author used an inflow turbulence generation technique based on an unsteady flow simulation method in the frame of a train moving coordinate system. In the case where the train speeds are $10 \mathrm{~m} / \mathrm{s}, 5.8 \mathrm{~m} / \mathrm{s}, 1.8 \mathrm{~m} / \mathrm{s}$ and $0 \mathrm{~m} / \mathrm{s}$ and the wind speed $i s 10 \mathrm{~m} / \mathrm{s}$ in the direction perpendicular to the train running direction, the pressure coefficient distribution on the surface of the running train was obtained by numerical simulation and compared with that from experimental studies. The detailed flow fields around the running train were also presented. Based on the comparison between the running train simulation and stationary train simulation with the same relative wind angles to the train, the running effects on aerodynamic characteristics in the case of the simple train model were discussed.
\end{abstract}

Keywords: large-eddy simulation, turbulent boundary layer, railway vehicle, crosswind

\section{Introduction}

There are only a few studies about the effects of train running on the aerodynamic characteristics in the research field of crosswind problems in railways, for example reference [1]. As a recent experimental research on the running effects, there has been a wind tunnel experiment using a moving model rig [2], where pressure distribution around the car is measured in detail. On the other hand, as a numerical research on the running effects, there has been a numerical simulation of flow around a train running through crosswind where inflow conditions do not simulate the turbulent velocity fluctuation, but simulate only the mean velocity gradient distribution [3]. With respect to numerical research, the validation of the numerical simulation was conducted for a wind tunnel measurement on a stationary train but not on a running train, because there was no available data at that time. After obtaining available experimental data by moving train rig, research of numerical simulation under the same conditions as those of the experiment including simulated inflow turbulence, was conducted in this research.

This paper describes the method of generation of the inflow turbulence to the flow field around the running train, conducting a numerical simulation under the same conditions as those in the wind tunnel experiment, comparison between the numerical simulation and the wind tunnel experiment, and comparison of the numerical results between a running train and a stationary one.

\section{Implementation of inflow turbulence to the simu- lation of flow around a running train}

\subsection{Computational model}

The target flow of numerical simulation in this re- search is a flow field around a train at the speed of $V$, running through crosswind speed $W$ of a yaw angle $\alpha$. Computation of the flow around the train in the unsteady wind (turbulent boundary layer) is conducted by parallel computation of the turbulent boundary layer and flow around the train. In the parallel computation, the result of instantaneous turbulent boundary layer simulation is used as the inflow boundary condition in the computation of the flow around the train.

The computational models of the turbulent boundary layer and the flow around a train are shown in Fig. 1. The shape of the train is a rectangular solid which is a simple imitation of one railway vehicle, with vehicle height $H$, width $1.061 H$, length $7.386 H$, and gap between car under floor and ground $0.398 H$. The dimensions selected here are the same as those in reference [2].

The coordinate system used in simulation of both the turbulent boundary layer and the flow around the train is fixed on the running train. Figure 1 shows the coordinate system fixed on the ground, but whole computational domains are moved with train speed $\boldsymbol{V}$ in the simulation. In other words, in the turbulent boundary layer simulation, wind $\boldsymbol{W}$ (with yaw angle $\alpha$ ) is not used, rather, relative wind against the train $\boldsymbol{U}=\boldsymbol{W}-\boldsymbol{V}$ (with yaw angle $\beta$ ) is used. As $W$ varies depending on the height from the ground, the relative wind against the train $U$ and yaw angle $\beta$ are varied depending on the height as shown in Fig. 2.

The turbulent boundary layer simulation (Figure 1 (a)) uses a uniform computational grid in the $x$ and $z$ directions $(\Delta x=\Delta z=0.25 H)$ and a non-uniform grid in the $y$ direction $(\Delta y=0.01-1.00 H)$. The total number of computational grid points is one million. The simulation of the flow around the train (Figure 1 (b)) uses a computational grid where the minimum grid space is $0.01 H$ (near the ground and train surface). The total number of computational grid points is 13 million. 


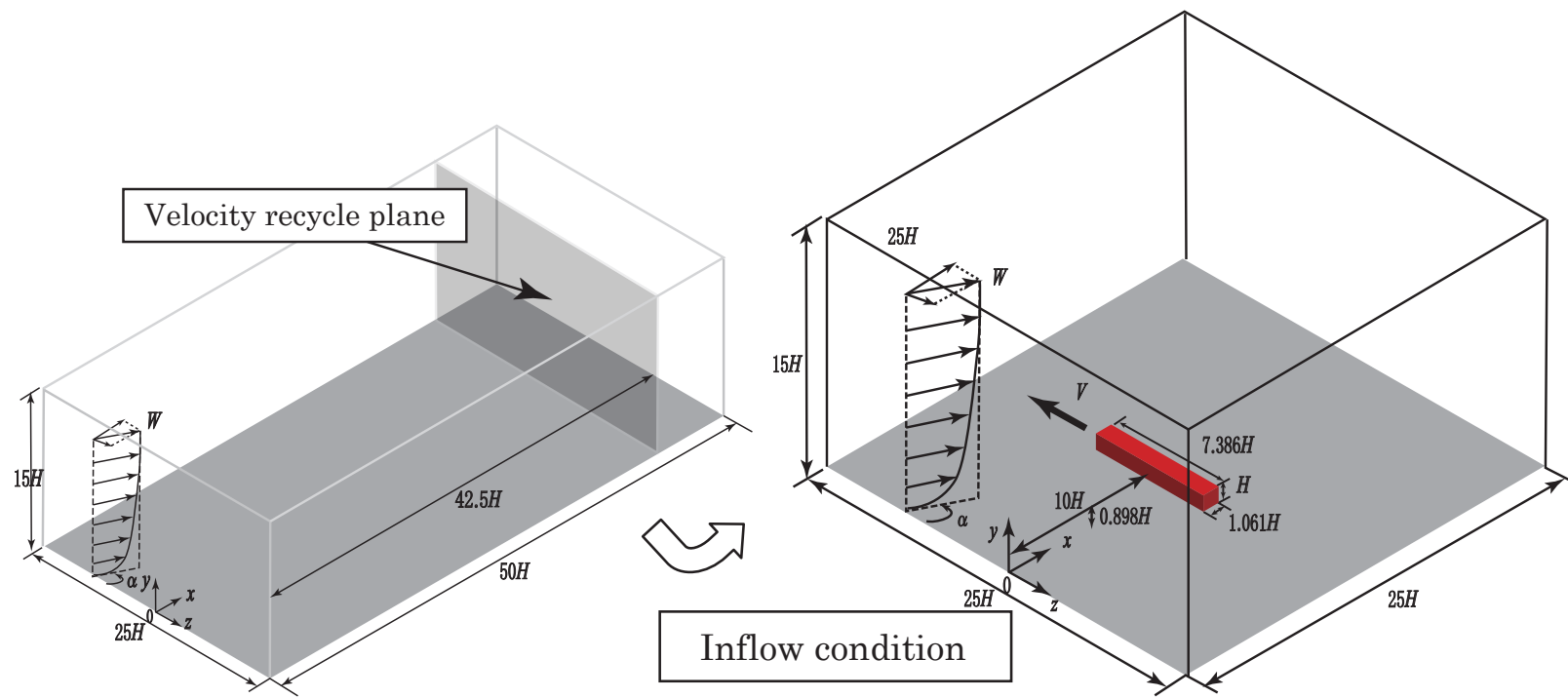

(a) Simulation of turbulent boundary layer

(b) Simulation of flow around train

Fig. 1 Computational model
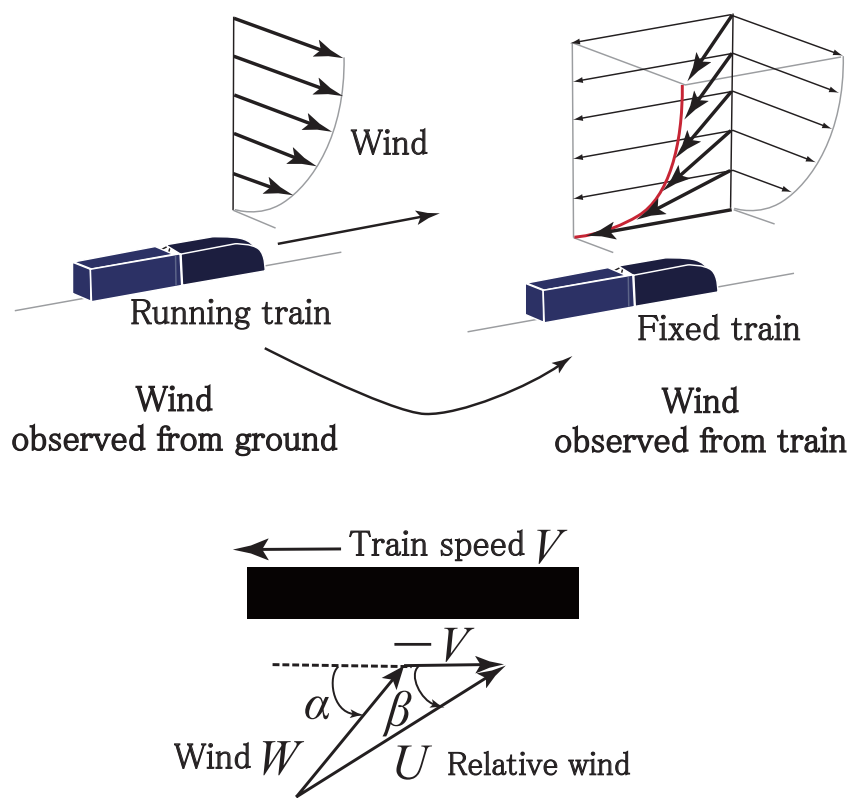

Fig. 2 Wind observed from running train

\subsection{Computational method for the turbulent bound- ary layer}

The computational method for the turbulent boundary layer is the same as the previous method which was used in the simulation of a flow above an embankment [4] referring to the method of Kataoka et.al [5]. In this method, supposing that the space development of the turbulent boundary layer is small, the inflow boundary condition flow is determined by the flow in the velocity recycle plane which is far from the inflow plane.

The computation in this research needs new techniques to realize the yaw angle of wind and compute flows in the train moving coordinate system. These requirements were solved by extending the above computational method for the boundary layer to the arbitrary inflow yaw angle and coordinate system, which were investigated in this research.

The mean velocity distribution in the inflow plane was determined by the wind profile of the power law with the exponent of $1 / 7$ and the boundary layer thickness $\Delta=2.9 \mathrm{H}$ with reference to the wind tunnel test [2].

\subsection{Boundary conditions}

The boundary conditions in the simulation of the turbulent boundary layer shown in Fig. 1(a) are as follows: the inflow boundary condition is given by the method in section 2.2; the outflow boundary condition is the convective outflow condition; the ground speed is fixed at $-\boldsymbol{V}$; the velocity far from the ground is fixed at the uniform flow of the turbulent boundary layer; and the periodic condition is used in the span direction ( $z$ direction).

The boundary conditions in the simulation of the flow around the train shown in Fig. 1(b) are as follows: the inflow boundary condition is given by the instantaneous velocity of the turbulent boundary layer in the recycle plane shown in Fig. 1(a); the outflow boundary condition is the convective outflow condition; the ground speed is fixed at $-\boldsymbol{V}$; the velocity condition far from the ground velocity uses the slip condition; and the periodic condition is used in the span direction ( $z$ direction).

\subsection{Numerical method}

Three-dimensional filtered incompressible NavierStokes equations are used as governing equations. The dynamic Smagorinsky model is used with the least square technique. The conservative higher order finite difference schemes are used to discretize the governing equations, which are advanced in time by the fractional-step method. In this study, the author used the fourth order schemes for space discretization, and the third order Adams-Bashforth 
method and Crank-Nicolson method for time discretization. To prevent numerical instability, forth-order numerical dissipation was added in the convective term.

The Reynolds number $W_{0} H / v$ used in the simulation, ( $W_{0}$ : uniform flow of turbulent boundary layer of the wind $W$, $H$ : height of the train, $v$ : kinematic viscosity) was determined to be 29,000 with reference to the wind tunnel test [2], whose Reynolds number was small compared with that of the real situation (the order of $10^{6}$ ). The time increment $\Delta T$ was $0.001 H / W_{0}$, whose CFL number was about 0.1-0.2. The computation was implemented up to the time $T=200 \mathrm{H} /$ $W_{0}$, and the mean value was calculated by using the data from $T=50 H / W_{0}$ to $T=200 H / W_{0}$, which was equivalent to 0.66 second in the case of $W_{0}=10 \mathrm{~m} / \mathrm{s}$ and $H=0.044 \mathrm{~m} \mathrm{(1/60} \mathrm{scale}$ model). Before starting the parallel simulation of the turbulent boundary layer and the flow around the train, the single simulation of the turbulent boundary layer was conducted until the statistically steady condition of the flow field was realized to reduce computational time.

\subsection{Computational conditions}

The train height $\mathrm{H}$ is assumed to be $0.044 \mathrm{~m}$, and the uniform flow of the turbulent boundary layer of the wind W0 is assumed to be $10 \mathrm{~m} / \mathrm{s}$. The first simulation calculation was executed for a turbulent boundary layer with the yaw angle shown in section 2.6, where the yaw angles $\alpha$ are 90,70 , and 45 degrees. The second simulation calculation was executed for the running train shown in the section 3 , where the train speed are $0,1.8,5.8$, and $10 \mathrm{~m} / \mathrm{s}$. The third simulation calculation was executed for the stationary train to be compared with the running train in section 4 , where the yaw angles $\alpha$ are 40.6, 56.2, and 78.1 degrees. Those yaw angles were determined to be $\beta_{c}$, the same as the angle at mid-height on the running train car.

\subsection{Computation of the turbulent boundary layer with the yaw angle}

In this section the results of the computation of the turbulent boundary layer with varying yaw angles demonstrably validate the computational method developed in this research. The mean velocity distribution and the stan-

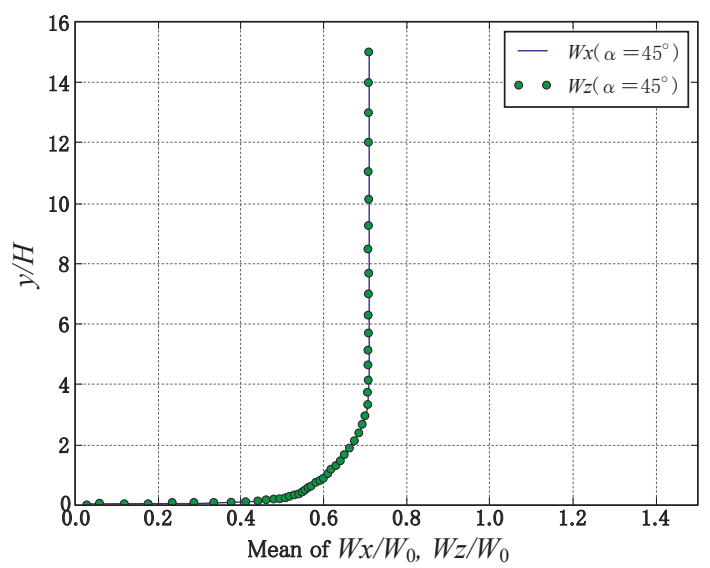

(a) Mean velocity distribution dard deviation of the velocity distribution at the position $x=10 H$ in the computational domain of the turbulent boundary layer are shown in Fig. 3. Here $x=10 H$ corresponds to the position of the windward surface of the train during simulation of the flow around the train. The result of the mean velocity distribution shows that the velocity depending on yaw angle is appropriate by the result obtained with $W_{x}=W_{z}$ for a yaw angle $\alpha=45$ degrees as shown in Fig. 3(a). The result of the standard deviation of velocity distribution in the case of yaw angle $\alpha=90$ degrees shows that the standard deviation in the flow direction is larger than in the span direction, which is well known about the general turbulent boundary layer. In addition, in the case of a yaw angle $\alpha=45$ degrees, the standard deviation of velocity $W_{x}$ is the same as for $W_{z}$, and in the case of a yaw angle $\alpha=70$ degrees, the magnitude of the standard deviation of velocity is between $\alpha=90$ degrees and 45 degrees as shown in Fig. 3(b). From the above discussion, the author concluded that the turbulent boundary layer method developed in this work was validated.

\section{Comparison of numerical simulations and wind tunnel experiments with running train}

In this section, the results of computation of the flow around the running train under the turbulent boundary layer conditions of a uniform flow $W_{0}=10 \mathrm{~m} / \mathrm{s}$ and wind angle $\alpha=90$ degrees and train speed conditions $V=0,1.8,5.8$, and $10 \mathrm{~m} / \mathrm{s}$ are explained.

As an example of the simulation of the flow around the running train in the turbulent boundary layer, the instantaneous flow fields for a train speed $V=5.8 \mathrm{~m} / \mathrm{s}$ are shown in Fig. 4. Figure 4(a) shows the velocity vectors in the horizontal plane at car mid-height (i) and in the vertical plane near the head of the car (ii), in the vertical plane at half the length of the car (iii), and in the vertical plane near the tail of the car (iv). From Fig. 4 it is observed that the train is exposed to a relative angled wind, that flow around the train is detached from the head, and that there are turbulence flows in the downstream from the train. Figure 4(b) shows the isosurface of pressure where red color indicates high pressure and blue color indicates low pressure. From

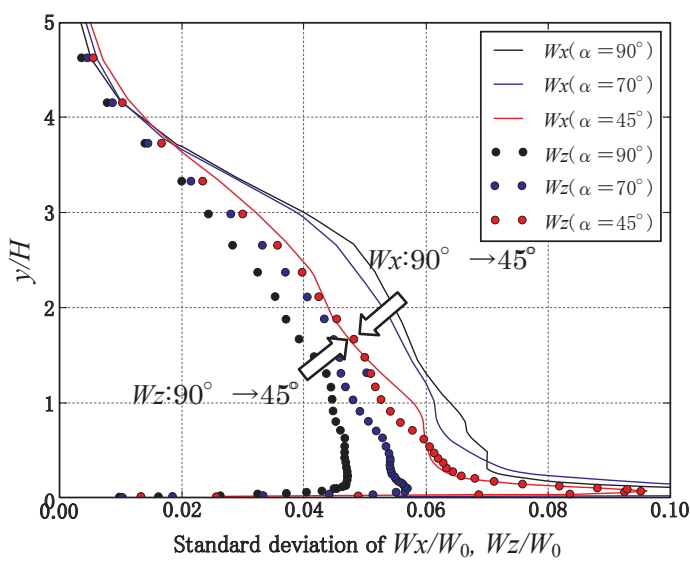

(b) Standard deviation of velocity

Fig. 3 Computational results of turbulent boundary layer with yaw angle 


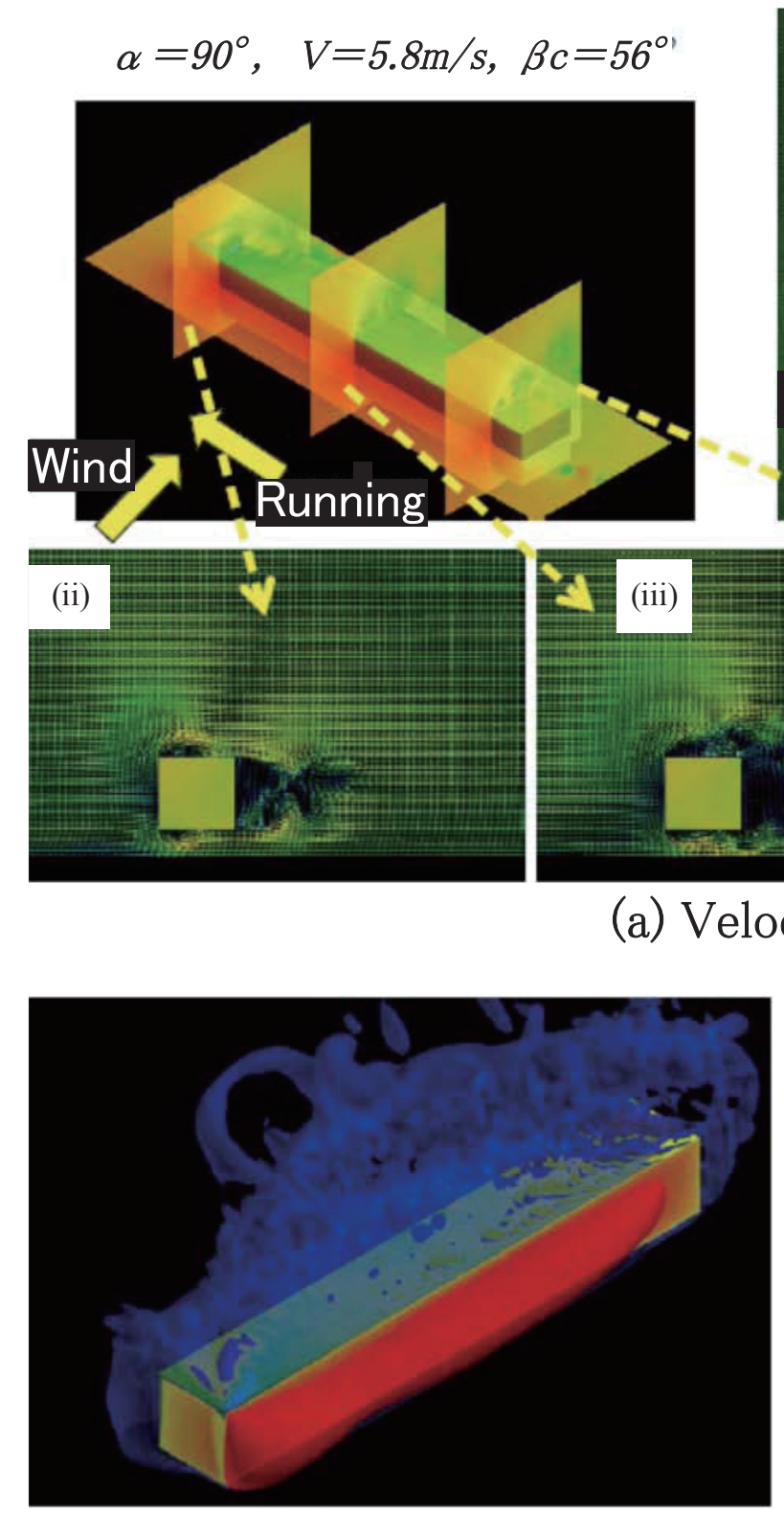

(b) Pressure contour
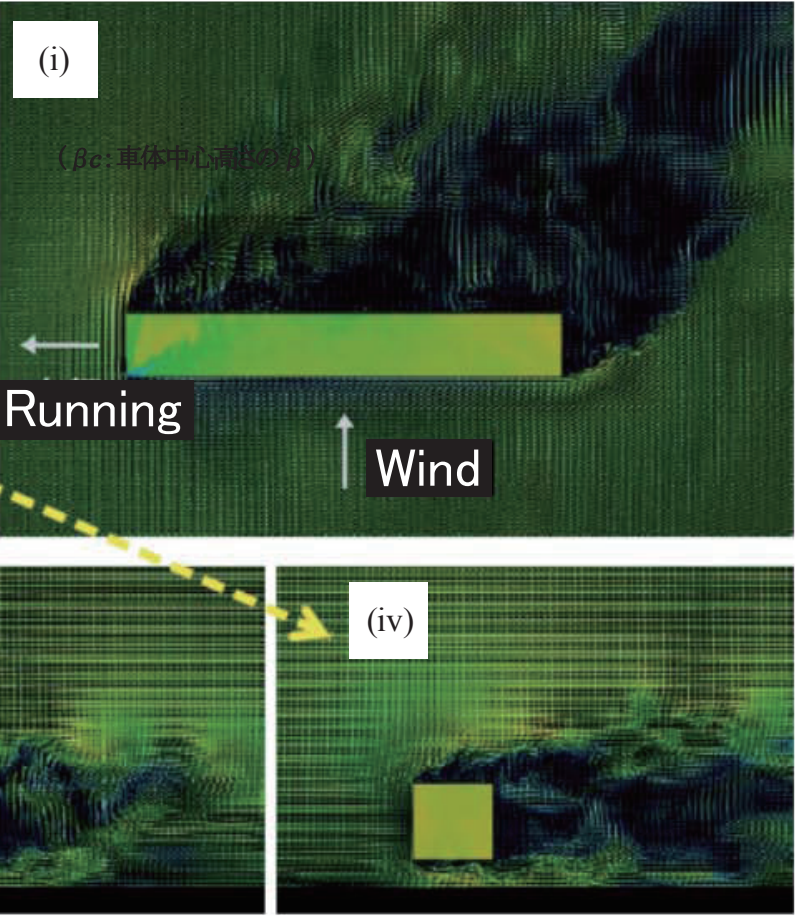

ity vector

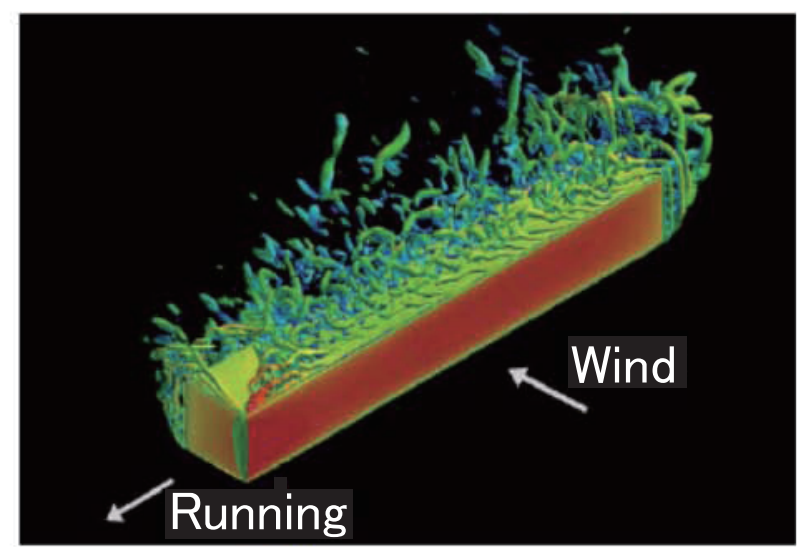

(c) Vortex structure

Fig. 4 Numerical simulation of flow around running train in turbulent boundary layer (instantaneous flow field)

Fig. 4(b) it is observed that on the windward side, the train has high pressure and that there is low pressure fluctuation in the flow separation region. Figure 4(c) shows the visualization of vortices structure using the isosurface of the second invariant of velocity gradient tensor. From Fig. 4(c) it is observed that complex vortex tubes exist and that one pair of vortex cones exists on the roof of the head of the car.

Figure 5 shows the comparison of the mean velocity and turbulence intensity distribution between the numerical simulation and the wind tunnel experiment at position $x=10 H$ when train speed is $0 \mathrm{~m} / \mathrm{s}$. Here, the reference velocity which creates a dimensionless form of turbulence intensity is the mean velocity at the same point of the turbulence, being different from the reference velocity used in Fig. 3(b) which is the uniform velocity out of the boundary layer. From Fig. 5 it is confirmed that there is a good agreement of the mean velocity and the turbulence intensity between the numerical simulation and the wind tunnel experiment. Figure 6 shows the comparison of the mean pressure distribution around the train on the horizontal plane at mid-height of the train between the numerical simulation and the wind tunnel experiment. It is confirmed that there is a good agreement between the numerical simulation and the wind tunnel experiment. 

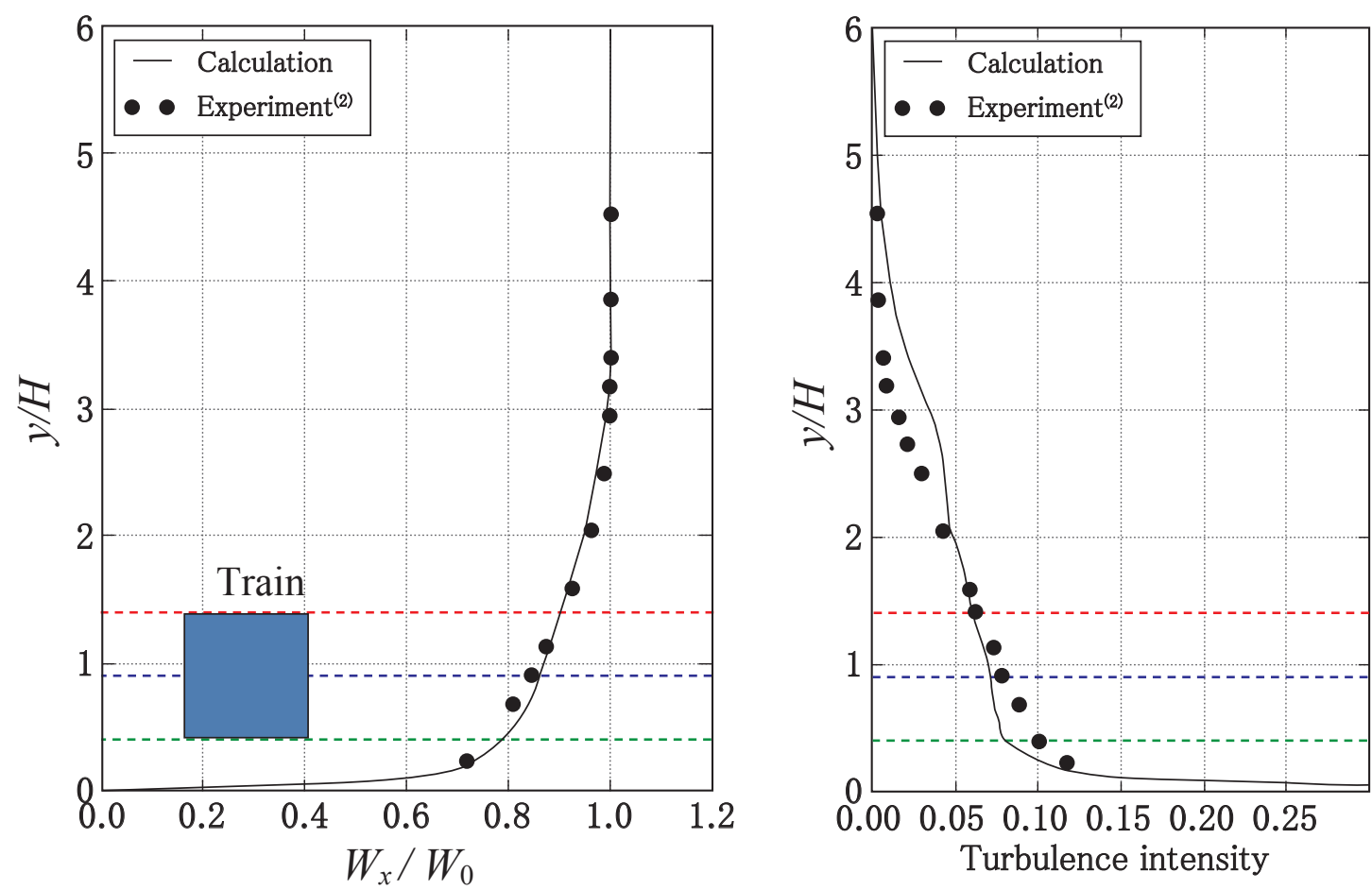

Fig. 5 Mean velocity and turbulence intensity in streamwise direction

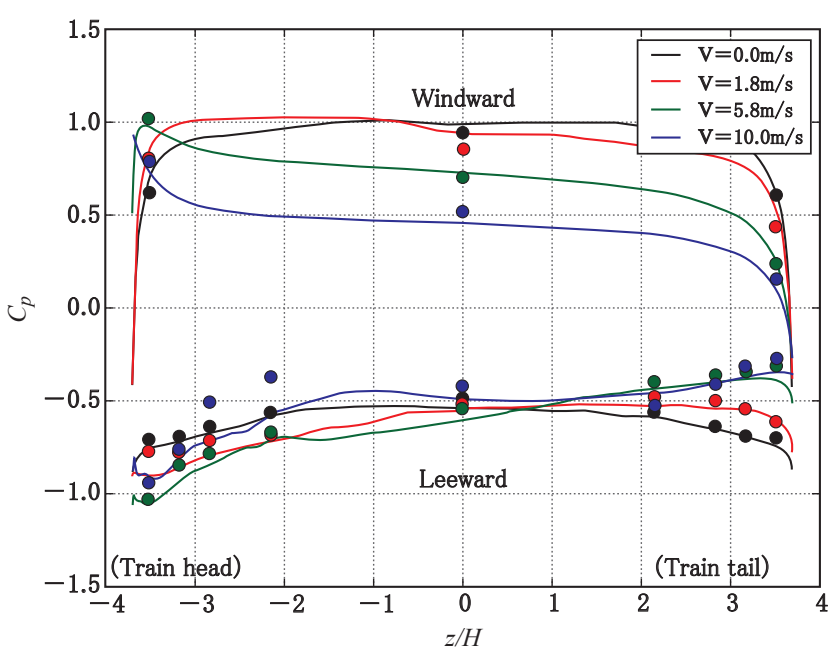

Fig. 6 Pressure coefficient distribution around train (symbol: calculation, line: experiment)

\section{Comparison of aerodynamic characteristics be- tween a running train and a stationary train}

Conventional wind tunnel experiments for an estimation of aerodynamic characteristics of a train under crosswind use stationary train models with an inflow wind angle which is equal to the relative yaw angle $\beta_{c}$ affecting the running train at mid-height of the train. In this section, the conventional wind tunnel experiment using a stationary train model is validated by numerical simulation. The train running effects on the aerodynamic characteristics are investigated by comparing numerical simulation results of a running train and a stationary train whose relative wind angles are the same. The wind angle $\alpha$ in the simulation of a stationary train is determined to be the same as the relative wind angle $\beta_{c}$ for the simulation of a running train.

Figure 7 shows the result of the comparison of the mean side force coefficient and the lift one, which are made dimensionless form by using relative wind velocity at the position of the middle of the train height and the side area of the train, between running train simulation and stationary train one. Figure 7 demonstrates that there is little difference between a running train and a stationary train. Figure 8 shows the comparison of the mean pressure distribution, which is made into a dimensionless form by using relative wind velocities at mid-height of the train. Figure 8 shows that there is little difference between a running train and a stationary train when using the simplified train model over flat terrain. These numerical simulation results are consistent with the results of wind tunnel experiments.

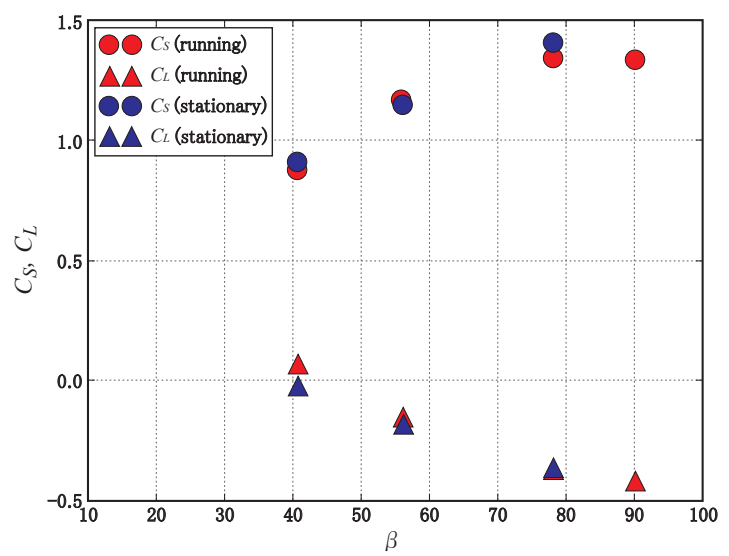

Fig. 7 Side force coefficient $C_{S}$ and lift coefficient $C_{L}$ 


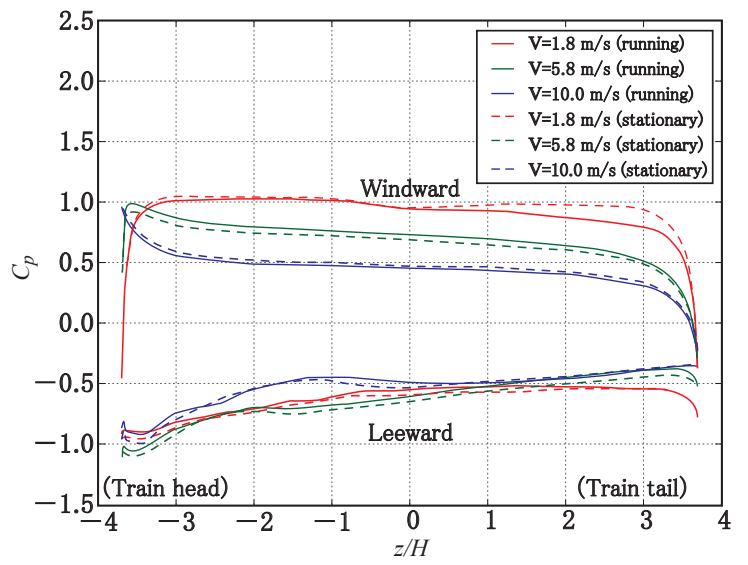

Fig. 8 Pressure coefficient distribution around train (solid line: running train, dashed line: stationary train)

\section{Conclusions}

The author conducted numerical simulations of flows around a running train in a turbulent boundary layer on a flat terrain. An inflow turbulence generation method was developed in this research by extending previous methods adopted for the numerical simulation of flows above a stationary structure to the coordinate system, set up for a train moving frame. The above method could be used to simulate both train running and inflow turbulence conditions. It was confirmed that the pressure distribution around the train in running train simulations agreed well with results from wind tunnel experiments on a moving train. When the same conditions are used for wind relative yaw angle, on comparison it was shown that the pressure distribution around the train, mean side force and lift coefficients for a running train and a stationary train differed little. The final results of the numerical simulation indicated that the train running effects on the aerodynamic characteristics of the simplified shape model over flat terrain was small.

\section{References}

[1] Baker, C.J., "Train aerodynamic forces and moments from moving model experiments," Journal of Wind Engineering and Industrial Aerodynamics, Vol.24, pp.227251, 1986.

[2] Suzuki, M., "Wind tunnel test of railway vehicle under cross wind using moving model rig," Wind Engineers, JAWE, Vol.36, No.3 (No.128), pp.258-263, 2011 (in Japanese).

[3] Nakade, K. and Suzuki, M., "Numerical simulation of flow around a running train under side winds," Fluids Engineering Conference, JSME, pp.599-600, 2009 (in Japanese).

[4] Nakade, K. and Suzuki, M., "Numerical simulation of flow around an embankment under side wind," RTRI Report, Vol.24, No.9, pp.51-56, 2010 (in Japanese).

[5] Kataoka H., and Mizuno M., "Numerical flow computation around 3d square cylinder using inflow turbulence," Journal of Architecture and Planning, 523, pp.71-77, 1999 (in Japanese).

[6] Kajishima, T., Ohta, T., Okazaki, K., and Miyake, Y., "High-order finite difference method for incompressible flows using collocated grid system," Transactions of the Japan Society of Mechanical Engineers, Series B, Vol.63, No.614, pp.3247-3254, 1997 (in Japanese).

[7] Germano, M., Piomelli, U., Moin, P., and Cabot, W.H., "A dynamic subgrid-scale eddy viscosity model," Physics of Fluids, A3-7, pp.1760-1765, 1991.

[8] Lilly, D.K., "A proposed modification of the Germano subgrid-scale closure model," Physics of Fluids, A4-4, pp.633-635, 1992.

\section{Authors}

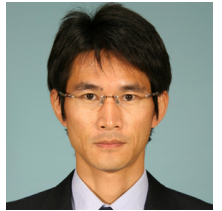

Koji NAKADE

Senior Researcher, Vehicle Aerodynamics

Laboratory, Environmental Engineering

Division

Research Areas: Fluid Mechanics,

Computational Fluid Dynamics 